\title{
Polymersomes and Wormlike Micelles Made Fluorescent by Direct Modifications of Block Copolymer Amphiphiles
}

\author{
Karthikan Rajagopal, David A. Christian, Takamasa Harada, Aiwei Tian, \\ and Dennis E. Discher \\ Department of Chemical and Biomolecular Engineering, University of Pennsylvania, Philadelphia, PA 19104, USA \\ Correspondence should be addressed to Karthikan Rajagopal, kr@seas.upenn.edu
}

Received 23 December 2009; Accepted 20 April 2010

Academic Editor: Jinying Yuan

Copyright (c) 2010 Karthikan Rajagopal et al. This is an open access article distributed under the Creative Commons Attribution License, which permits unrestricted use, distribution, and reproduction in any medium, provided the original work is properly cited.

Wormlike micelles and vesicles prepared from diblock copolymers are attracting great interest for a number of technological applications. Although transmission electron microscopy has remained as the method of choice for assessing the morphologies, fluorescence microscopy has a number of advantages. We show here that when commercially available fluorophores are covalently attached to diblock copolymers, a number of their physicochemical characteristics can be investigated. This method becomes particularly useful for visualizing phase separation within polymer assemblies and assessing the dynamics of wormlike micelles in real time. Near-IR fluorophores can be covalently conjugated to polymers and this opens the possibility for deep-tissue fluorescence imaging of polymer assemblies in drug delivery applications.

\section{Introduction}

In aqueous solution, amphiphilic diblock copolymers can spontaneously self-assemble to form supramolecular morphologies such as spherical and worm-like micelles and vesicles (polymersomes) [1-3]. Due to their unique physicochemical characteristics these polymeric assemblies have shown potential for various applications such as drug delivery. Wormlike micelles ("worms") increase the delivered dose of water-insoluble drugs such as Taxol $[4,5]$, and vesicles (polymersomes) can be used for the encapsulation and delivery of both water-insoluble (Taxol) and watersoluble therapeutics that range from drugs to proteins [6] to siRNA [7]. An understanding of morphologies from amphiphilic diblock copolymers in dilute solution as well as their characteristics would facilitate the generation of even more robust and useful supra-molecular materials. Seminal experiments of Eisenberg and Bates helped to show that classic ideas of surfactant-based morphologies also apply to block copolymers upon varying the relative sizes of the two dissimilar blocks $[8,9]$. While such studies used TEM to visualize frozen nano-morphologies, we have emphasized and exploited fluorescence imaging, including the use of dyes covalently conjugated to block copolymers as described here. Fluorescence microscopy helps to clarify many of the unique physical characteristics of polymer assemblies, including the diversity in sizes, shapes, flexibilities and lateral segregation.

Fluoroscence microscopy is widely used in cell biology and biophysics for understanding the structure and function of molecules within cells and organelles [10-12]. Although one cannot infer structural information below the diffraction limit, this method nevertheless enables the visualization of spatial and temporal evolution of processes within the cell. As a result, numerous fluorescent dyes have become commercially available for ready conjugation to biomolecules [13]. A number of these fluorophores have been specifically designed for aqueous phase reaction with various functional groups on amino acid side chains such as thiols, amines, hydroxyls and acids. On the other hand, TEM is particularly useful for identifying structures down to nanometer length scale and therefore has remained the method of choice for assessing the morphologies of polymer assemblies [14-17]. However, TEM imaging is performed on samples after drying, which can be an issue with water-based assemblies, and while 
cryo-TEM of vitrified samples partially circumvents the issue, the films are nano, contrast can be problematic, and information on dynamics of these structures in solution is simply not obtainable. Equipment costs, sample preparation complexities, and specialized training all limit the utility of TEM. Alternatively, fluorescence microscopy is a robust and simpler method that enables rapid assessment of structures as well as the dynamics of polymeric assemblies in real time. Because wormlike micelles and polymersomes formed from diblock copolymers are often microns in size, they can be easily identified and quantified using fluorescence microscopy-although it should be stated at the outset that one cannot unequivocally decipher differences between spherical micelles and submicron sized worm micelles and vesicles.

Visualization of polymer assemblies by fluorescence microscopy can be accomplished by adding a small amount of lipophilic dye (e.g., PKH26, Sigma) to a solution of the polymer sample. Such a dye molecule immediately partitions into the hydrophobic core of the polymer assemblies and therefore the shapes of the assemblies can be directly observed under the microscope. Using this approach, we have extensively characterized the physical properties and degradation of polymeric worm-like micelles and vesicles [18-22]. While these studies demonstrate that the externally added dye is minimally perturbing to the polymeric structures, their utility in some instances seems limited. For example, chemical incompatibility between the hydrophobic core and dye molecules may result in poor labeling and in some cases the dye molecule has been shown to leach out [23] or transfer to a different environment such as cell membranes [24]. We envisioned that covalent conjugation of a fluorophore to diblock copolymers may not only overcome some of these limitations, but it may also be useful, as we show here, in investigating phase separation within polymer assemblies as well as tracking their intracellular transport for drug delivery applications. We show here that several commercially available dyes can be readily conjugated to diblock copolymers using established chemistries, and when the modified polymers were blended with diblock copolymers in small proportions, their morphologies are retained and dynamics in real time can be observed using fluorescence microscopy. Finally, we show that near-IR emissive fluorophores can be attached to diblock copolymers and this will be particularly important for in vivo tracking of these assemblies via deep-tissue fluorescence imaging.

\section{Experimental Methods}

2.1. Materials. Polyethyleneoxide-b-polybutadiene (PEO-bPBD; 10,500 g/mole), polyacrylic acid-b-polyethyleneoxide (PEO-b-PAA; 10,500 g/mole), polycaprolactone-b-polyethyleneoxide (PCL-b-PEO; 15,500 g/mole), and polycaprolactone (PCL-OH; 7,700 g/mole) were obtained from Polymer Source, Canada. Thiol-terminated polycaprolactone (PCL-SH) was synthesized as described before from hydroxyl-terminated polycaprolactone (PCL-OH). Fluorescent dyes, cascade blue ethylenediamine trisodium salt (C621), fluorescein-5-carbonyl azide diacetate (F6218), rhodamine maleimide (R6029), and Alexa fluor 750 C5 maleimide (A30459) were obtained from Molecular Probes, Eugene, OR and 3,3'-dioctadecyloxacarbocyanine perchlorate $(\mathrm{DiO})$ was obtained from Sigma.

2.2. Conjugation of Fluorescein Azide Carboxylate to $P B D-b$ $P E O$. In a $5 \mathrm{~mL}$ RB flask $30 \mathrm{mg}\left(2.86 \times 10^{-3}\right.$ mMoles $)$ of PBD-b-PEO $(10,500 \mathrm{~g} / \mathrm{mole})$ and $6.9 \mathrm{mg}\left(14.3 \times 10^{-3} \mathrm{mM}\right)$ of fluorescein azide carboxylate $(485.41 \mathrm{~g} / \mathrm{mole})$ were taken and dissolved in $1.0 \mathrm{~mL}$ anhydrous toluene. The contents were stirred overnight on an oil bath at $85^{\circ} \mathrm{C}$. The reaction was stopped next day and $1.0 \mathrm{~mL}$ of anhydrous DMF and $200 \mu \mathrm{L}$ of hydroxylamine (50\%) were added to the flask. The contents of the flask turn dark orange and after stirring for 2 hours were transferred to a Spectrapor dialysis membrane (MWCO 3500) and dialysed against DMSO for 2 hours. This was followed by dialysis against DI water for two days and water was changed every 12 hours. The dye-conjugated polymer in dry form was recovered by freeze-drying.

2.3. Conjugation of Cascade Blue to PBD-b-PAA. To a solution of polybutadiene-b-polyacrylic acid (PBD-b-PAA) polymer ( $\left.10 \mathrm{mg}, 0.905 \times 10^{-3} \mathrm{mMoles}\right)$ in anhydrous DMF $(0.7 \mathrm{~mL}), 3$ equivalents of $\operatorname{EDAC}\left(0.52 \mathrm{mg}, 2.7 \times 10^{-3}\right.$ mMoles $)$ and $\mathrm{HOBt}\left(0.42 \mathrm{mg}, 2.7 \times 10^{-3}\right.$ mMoles $)$ were added and the contents were stirred under argon for 1 hour. A solution of cascade blue etylenediamine $(1.7 \mathrm{mg}$, $\left.625 \mathrm{~g} / \mathrm{mole}, 2.7 \times 10^{-3} \mathrm{mMoles}\right)$ in anhydrous DMF $(0.3 \mathrm{~mL})$ was prepared separately and added to the reaction mixture. The contents were stirred for 36 hours and the dyeconjugated polymer was separated by dialysis against Millipore water (18.2 M $\omega . \mathrm{cm})$ using a Slide-A-Lyzer (Thermo Scientific) dialysis cassette (MWCO:10 kD). After 4 days of dialysis, the aqueous polymer solution was frozen and lyophilized to yield the dry polymer conjugated with the dye. To remove any residual and unreacted dye, the polymer was further washed with water and methanol and dried under vacuum. Covalent conjugation was established by thin layer chromatography using methanol as eluent.

2.4. Conjugation of Maleimide Dyes to PCL-SH. Thiolterminated polycaprolactone (PCL-SH) $\quad(7700 \mathrm{~g} / \mathrm{mole}$, $36 \mathrm{mg}, 4.7 \times 10^{-3}$ mMoles) was taken in a clean $5 \mathrm{~mL}$ $\mathrm{RB}$ flask and dissolved in $0.7 \mathrm{~mL}$ of freshly distilled DCM. Separately 1.2 equivalents of rhodamine maleimide $\left(680.8 \mathrm{~g} / \mathrm{mole}, 3.82 \mathrm{mg}, 5.61 \times 10^{-3} \mathrm{mMoles}\right)$ was dissolved in $40 \mu \mathrm{L}$ of anhydrous DMSO and added to the RB flask. The contents were stirred overnight at room temperature under argon. The dye-conjugated polymer was separated by dialysing (Spectrapor membrane, MWCO: 3500) for a day against DCM followed by dialysis against DMSO for a day. The purified polymer in dry form was obtained after freeze-drying from DMSO solution. For the conjugation of Alexa Flour C5 750 maleimide (1350 g/mole), PCL-SH $\left(5.7 \mathrm{mg}, 7700 \mathrm{~g} / \mathrm{mole}, 0.74 \times 10^{-3} \mathrm{mMoles}\right)$ was dissolved in freshly distilled DCM in a $5 \mathrm{~mL}$ RB flask. Separately 1.0 equivalent of Alexa Flour dye $\left(1 \mathrm{mg}, 0.74 \times 10^{-3} \mathrm{mMoles}\right)$ 
was dissolved in $10 \mu \mathrm{L}$ DMSO and transferred to the flask containing PCL-SH. The contents were stirred overnight under argon. The dye-conjugated polymer was separated by dialysing (Spectrapor membrane, MWCO: 3500) against DCM for a day followed by dialyzing against DMSO for a day. The purified polymer in dry form was obtained after freeze-drying.

2.5. Preparation of Polymer Assemblies. Polymer assemblies were prepared using one of two methods. In the solvent evaporation method, a $1 \mathrm{mM}$ stock of the unlabeled and labeled polymer was first prepared in chloroform separately. In a $4 \mathrm{~mL}$ amber vial $95 \mu \mathrm{L}$ of the unlabeled polymer and $5 \mu \mathrm{L}$ of the labeled polymer were added and mixed well. To the vial $1 \mathrm{~mL}$ of DI water was added and the contents were stirred gently $(\sim 200 \mathrm{rpm})$ on a magnetic stir plate using a micro stir bar with the cap open at room temperature. Stirring was continued for 48 hours or until no chloroform layer was observed. The final polymer concentration after chloroform evaporation was $100 \mathrm{mM}$. In the film rehydration method, $100 \mu \mathrm{L}$ of $1 \mathrm{mM}$ polymer stock solution containing $5 \mu \mathrm{L}$ of labeled polymer was diluted to $800 \mu \mathrm{L}$ with chloroform in a $4 \mathrm{~mL}$ amber glass vial. Chloroform was evaporated by gently purging nitrogen into the vial until a thin film of the polymer was formed on the inner surface and the vial was further dried in vacuum for at least 8 hours. To the dried vial, $1 \mathrm{~mL}$ of DI water was added, placed in a preheated oven at $60^{\circ} \mathrm{C}$ with cap closed and gently stirred $(\sim 100 \mathrm{rpm})$ for 12 hours. Phase separated polymersomes were formed by film rehydration method using $0.05 \mathrm{mg}$ of polymer $(0.033 \mathrm{mg}$ of TMRCA labeled PBD-b-PEO, $0.015 \mathrm{mg}$ of unlabeled PBDb-PAA, $0.0017 \mathrm{mg}$ of cascade blue labeled PBD-b-PAA) hydrated with $500 \mu \mathrm{L}$ of DI water at $\mathrm{pH} 3.5$ and $0.1 \mathrm{mM}$ $\mathrm{Ca}^{2+}$.

2.6. Fluorescence Microscopy. Morphologies were assessed using epifluorescence microscopy on an Olympus IX71. In an Eppendorf tube $10 \mu \mathrm{L}$ of the sample after self-assembly was taken and diluted to $100 \mu \mathrm{L}$ with DI water. A small amount of sample $(\sim 3 \mu \mathrm{L})$ was spotted on a precleaned glass slide and $18 \mathrm{~mm}$ circular cover slip was placed on top and gently pressed so as to form a thin film of the sample. The circular end of the cover slip was sealed using vacuum grease. The sample was placed on the microscope stage and imaged through the cover slip. For time lapse imaging a sequence of images from the same field of view was captured at $4 \mathrm{~s}$ interval. Two color phase separated polymersomes were imaged by laser-scanning confocal microscopy on an Olympus Fluoview 300 with $405 \mathrm{~nm}$ and $543 \mathrm{~nm}$ laser excitation sources. Laser scanning confocal microscopy on an Olympus Fluoview 1000 was used to image AlexaFluor 750-labeled polymersomes with a $748 \mathrm{~nm}$ laser excitation source.

2.7. Intracellular Tracking of Fluorescent Polymersomes. Approximately 50,000 A549 human lung carcinoma cells were seeded on $35 \mathrm{~mm}$ Matek dish 24 hours prior to the addition of polymersome sample. Ham's F12 (Invitrogen) with $10 \%$ FBS and $1 \%$ penicillin/streptomycin was used for culturing A549 cells. After exchanging with fresh medium and adding the sample, cells were incubated 24 hours for PCL-b-PEO/PCL-Rhodamine and 12 hours for PCL-b$\mathrm{PEO} / \mathrm{DiO}$ at $37^{\circ} \mathrm{C}$ with $5 \% \mathrm{CO}_{2}$. At the end of incubation, Hoechst 33342 in PBS was added 5000-fold dilution. After 10-minute incubation with Hoechst 33342, cells were washed with PBS three times and fresh medium was added. Cells were observed under fluorescent microscope without fixation.

2.8. Near IR Imaging Using Licor. Assemblies labeled with the AlexaFluor 750 fluorophore were diluted 10 fold in PBS and dispensed into a 96 well plate. The 96-well plate containing these solutions was then imaged on a Licor Odyssey using the $800 \mathrm{~nm}$ laser excitation source.

\section{Results and Discussion}

3.1. Covalent Attachment of Fluorophores to Diblock Copolymers Polymers. Two different diblock polymers were chosen for covalent conjugation to fluorescent dyes. The reaction schemes for dye conjugation are presented in Scheme 1 and the details of fluorophores used are shown in Table 1. Polybutadiene-b-poly(ethylene oxide) (PBD-bPEO) diblock copolymer was made fluorescent by reacting the terminal hydroxyl group of the PEO block with fluorescein-5-carbonyl azide. In anhydrous organic solvents, alcohols react with isocyanates to form urethane linkage. However, due to the limited stability of isocyanates, acylazide bearing fluorophores that are converted to isocyanates in situ can be used to label hydroxyls [25, 26]. PBD-b-PEO was dissolved in anhydrous toluene along with fluorescein acyl azide and heated to $85^{\circ} \mathrm{C}$. The acyl azide first undergoes a Curtius rearrangement to form an isocyanate that subsequently reacts with the terminal hydroxyl on the PEO block to form a stable urethane linkage. The resulting product becomes fluorescent only after hydrolyzing the carbonyl ester using hydroxylamine.

Polybutabiene-b-polyacrylic acid (PBD-b-PAA) was made fluorescent by coupling an amine containing fluorescent dye, cascade blue ethylenediamine, to acid groups on PAA using EDAC chemistry. EDAC is a watersoluble reagent that is used for the activation of carboxylic acids for reaction to amines. Since the diblock copolymer is not directly soluble in water this reaction was performed in anhydrous DMF. The first step in the reaction is the formation of $\mathrm{O}$-acylurea intermediate that is relatively unstable and slow to react with amines. However, the stability of the intermediate and the yield of amide bonds can be enhanced by including hydroxybenzotriazole (HOBT) in the reaction. This results in the formation of HOBT ester that readily reacts with amines. In order to couple cascade blue ethylenediamine to PBD-b-PAA, 3 equivalents of dye was used resulting in efficient coupling as established using TLC. However, it is important to note that for fuorescence microscopy yield of the reaction is not of a major concern as long as one can image these assemblies. 


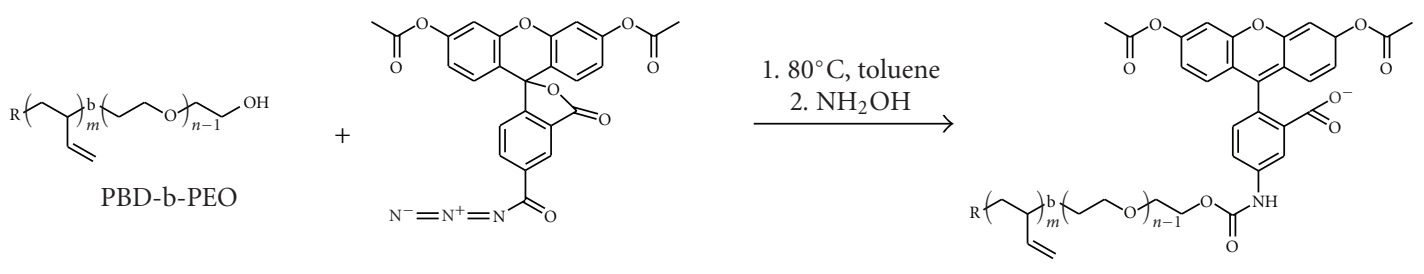

(a)

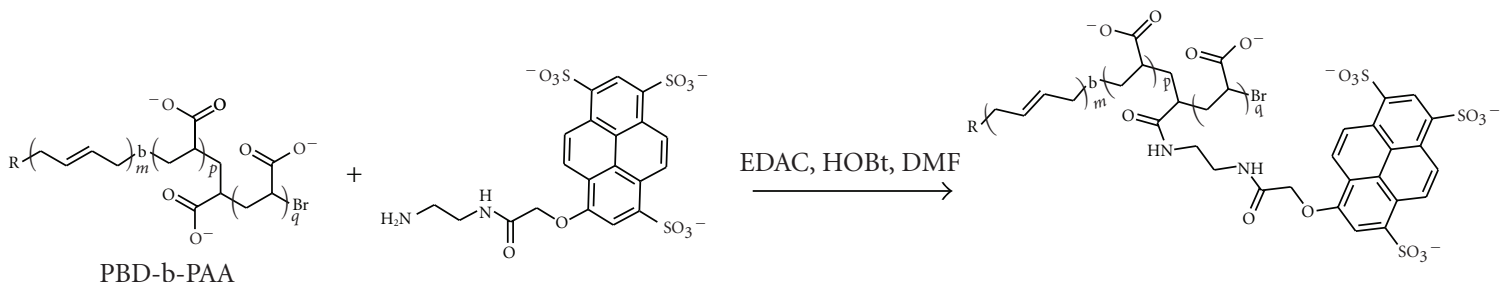

(b)

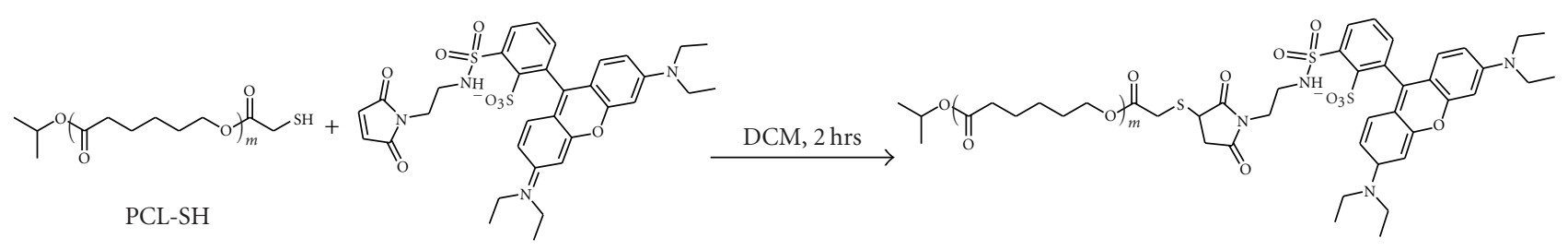

(c)

SCHEme 1: The conjugation of various reactive fluorophores to polymers is shown.

TABLE 1: Details of fluorophores used.

\begin{tabular}{lcccc}
\hline Fluorophore & MW (g/mole) & Absorption $(\mathrm{nm})$ & Extinction coefficient & Emission $(\mathrm{nm})$ \\
\hline Cascade blue ethylenediamine trisodium salt & 624.49 & 399 & 30,000 & \\
Fluorescein-5-carbonyl azide diacetate $^{\star}$ & 485.41 & 490 & $>70,000$ & 423 \\
Rhodamine maleimide & 680.8 & 560 & 119,000 & 510 \\
Alexa Fluor 750 C5-maleimide & 1350 & 753 & 290,000 & 580 \\
\hline
\end{tabular}

* Becomes fluorescent after hydrolysis of carbonyl group.

3.2. Fluorescence Microscopy Visualization of Polymer Assemblies. In order to visualize the morphologies by fluorescence microscopy, $5 \mathrm{~mol} \%$ of the labeled polymer was blended with the unlabeled polymer prior to self-assembly. The unlabeled and labeled polymers were first dissolved separately in chloroform, a good solvent for both the hydrophobic and hydrophilic blocks, and mixed prior to self-assembly. Selfassembly was initiated using one of two standard methods. In the first method, referred to as solvent evaporation, $100 \mu \mathrm{L}$ of the stock containing 5\% of labeled polymer was taken in a vial and hydrated by adding $1 \mathrm{~mL}$ of DI water at room temperature. Upon gentle stirring as the chloroform evaporates, the polymers spontaneously self-assemble. The second method used was standard film-rehydration, where $100 \mu \mathrm{L}$ of the polymer stock solution containing $5 \%$ of labeled polymer was added to a vial and diluted to $1 \mathrm{~mL}$ by adding chloroform and then cast into a thin film on the surface by drying. The vials were then hydrated at $60^{\circ} \mathrm{C}$ to induce self-assembly.
The morphologies obtained from the self-assembly of diblock copolymers were visualized directly using fluorescence microscopy. Figures 1(a) and 1(b) show vesicles formed from PBD-b-PAA and PBD-b-PEO diblock copolymers respectively. Labeling of the vesicle membrane gives vesicles an edge-bright fluorescence, although the membrane thickness is not resolvable by fluorescence microscopy. The edge brightness cannot be seen in vesicles with submicron diameters and thus they usually appear as bright spots. Since the amount of polymer incorporated within a spherical micelle is much less than that of a sub-micron sized vesicle, vesicles can be distinguished from spheres as they appear as much brighter structures. Nevertheless, This method allows us to identify the formation of multilamellar vesicles as shown in the left panel of Figure 2(a).

3.3. Blending PCL-Rhodamine for Imaging PEO-b-PCL Assemblies. The morphologies of PCL-b-PEO assemblies were visualized by blending in rhodamine conjugated to the 

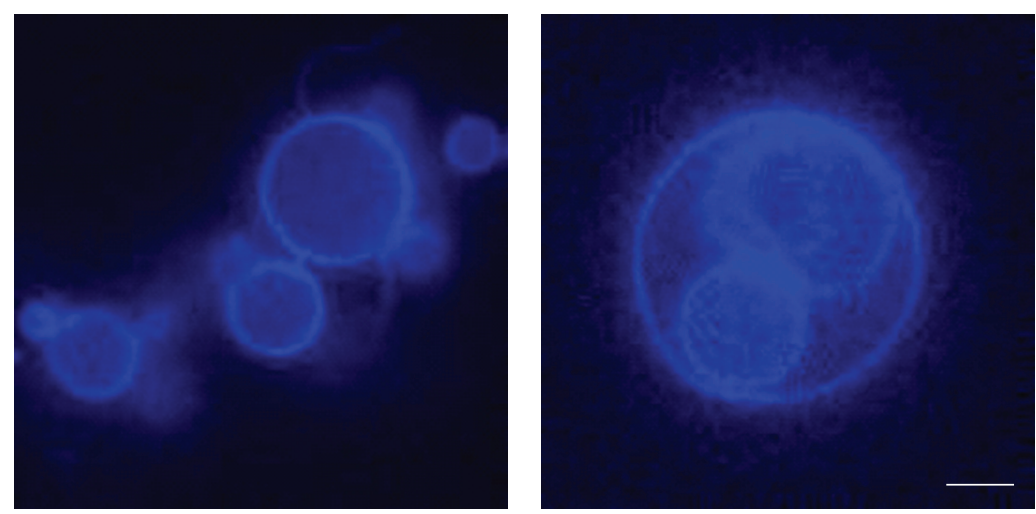

(a)
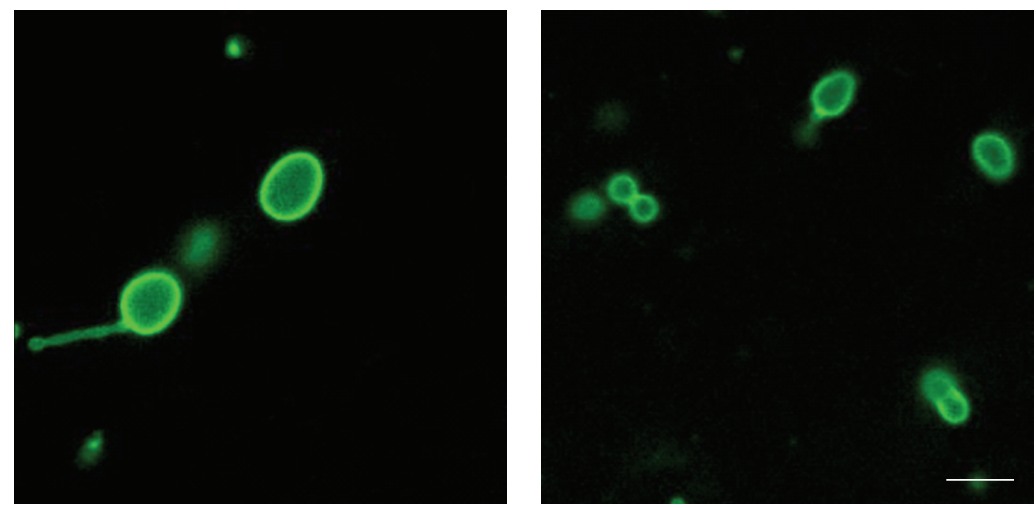

(b)

FIgURE 1: Morphologies from fluorescence microscopy. (a) Vesicles prepared from cascade blue conjugated to PAA-b-PBD. (b) Vesicles prepared from fluorescein conjugated to PBD-b-PEO. Scale bar is $5 \mu \mathrm{m}$.

polycaprolactone (PCL-Rh). Since PEO-b-PCL was synthesized by the ring-opening polymerization of $\mathcal{E}$-caprolactone, using $\mathrm{PEO}$ as the macroinitiator the resulting diblock copolymer does not have a reactive functional group on the PEO terminus for dye conjugation. Covalent attachment of dye to the hydroxyl terminus on the PCL side was not preferred, as it is likely to alter the geometry of the unimer, which might be perturbing to the morphology. In order to visualize these assemblies, a thiol-terminated polycaprolactone was conjugated to a maleimide bearing rhodamine and blended with PCL-b-PEO in small proportions. The reaction of thiols to maleimide is one of the common methods for labeling and attaching functional groups to proteins. The addition of a thiol to maleimide (Michael addition) results in the formation of a stable thioether bond and this reaction can be performed in aqueous phase at $\mathrm{pH}$ 7.0. However, since PCL is insoluble in water, the conjugation of maleimide rhodamine was performed using anhydrous DCM.

When chloroform solutions of PCL conjugated rhodamine and PCL-b-PEO were mixed, they co-self-assemble upon hydration and the resulting morphologies are presented in Figure 2(a). When 5\% of PCL-rhodamine is mixed with PCL-b-PEO, self-assembly results in the formation of a mixture of worm-like micelles and vesicles (Figure 2(b)). However, at 50\% PCL-rhodamine the dominant morphology is shifted to that of vesicles (Figure 2(c)). One of the major driving forces for self-assembly of amphiphilic diblock copolymers is the cohesive intermolecular hydrophobic interaction between PCL blocks. It is therefore not surprising to note that PCL-Rh is easily integrated within the hydrophobic cores of PCL-b-PEO assemblies.

3.4. Real Time Dynamics of Worm Micelles. Due to their "Brownian" interactions with water, worm micelles exhibit persistent thermal fluctuations along their contour lengths. The dynamics of worm micelles is clearly reflective of the physical state of the hydrophobic core-a rubbery or fluidic core gives rise to a flexible worm micelle. In this context, fluorescence microscopy is particularly attractive for assessing the fluctuation dynamics of polymer assemblies: the flexibilities of worm micelles can be readily quantified using time-lapse epifluorescence microscopy. This is shown in Figure 3(a) for a flexible PCL-b-PEO worm micelle incorporating 5\% PCL-rhodamine. At long-time intervals, the conformation of the worm micelle is fully decorrelated. However, within the same sample there is a small proportion of worm micelles that are completely inflexible and exhibit rigid body motion in solution, as shown in Figure 3(b). The rigidity of worm micelles is perhaps due to crystallization of polycaprolactone and is currently an active area of investigation. Although the melting temperature of PCL 

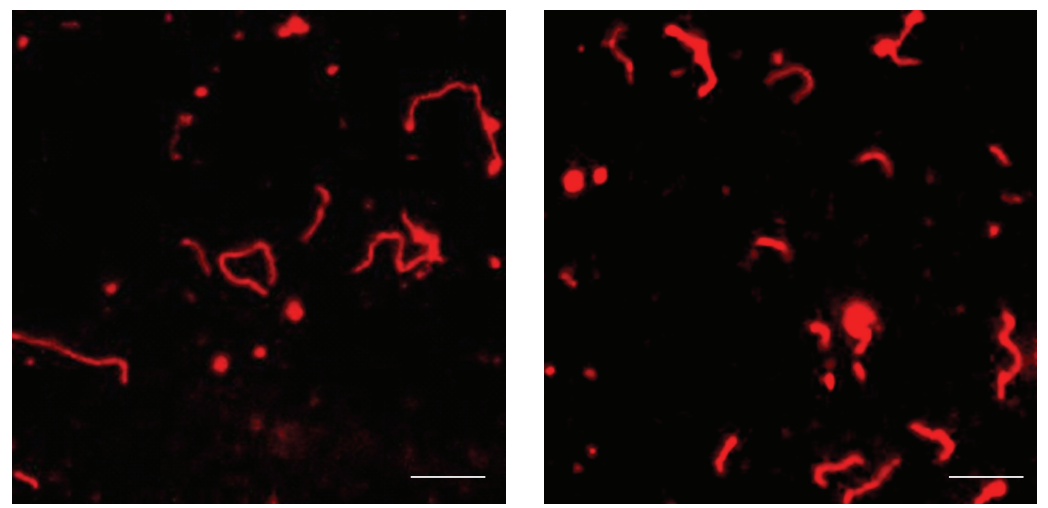

(a)

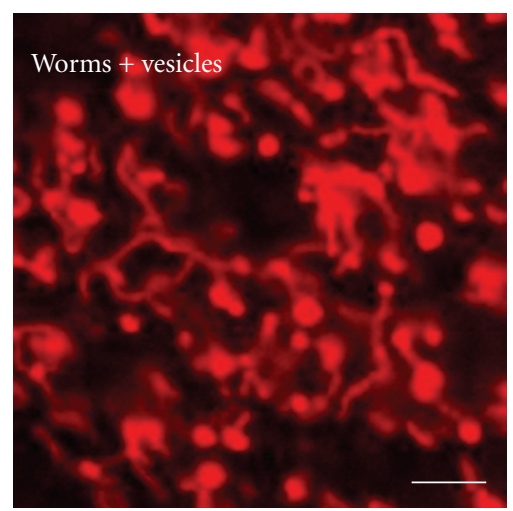

(b)

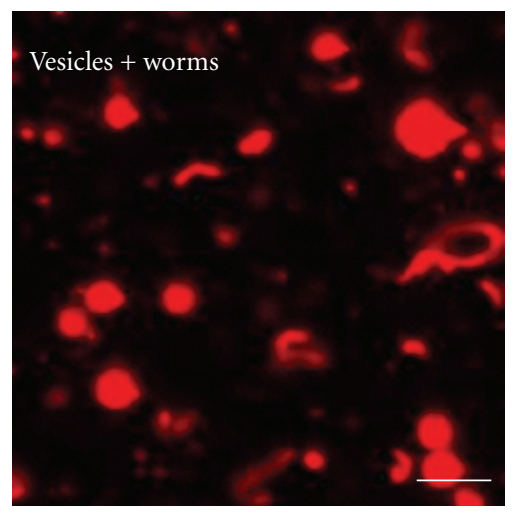

(c)

Figure 2: Blending PCL-Rhodamine in PCL-b-PEO assemblies. (a) Worm micelles and vesicles prepared from PEO-b-PCL diblock copolymer blended with 5\% PCL-rhodamine. (b) At 5\% PCL-rhodamine blending PCL-b-PEO worm micelles are obtained as the dominant morphology. (c) At 50\% PCL-rhodamine, the dominant morphology shifts to vesicles. Scale bar is $10 \mu \mathrm{m}$.
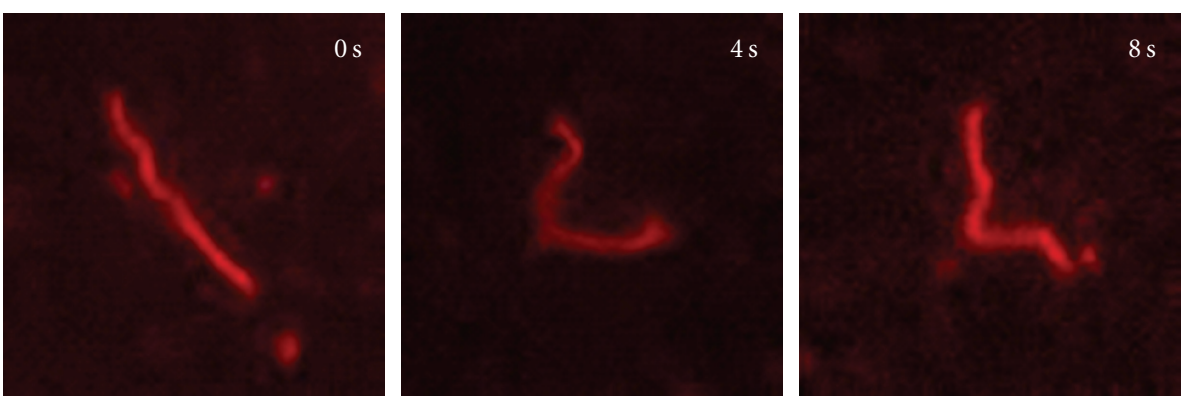

(a)
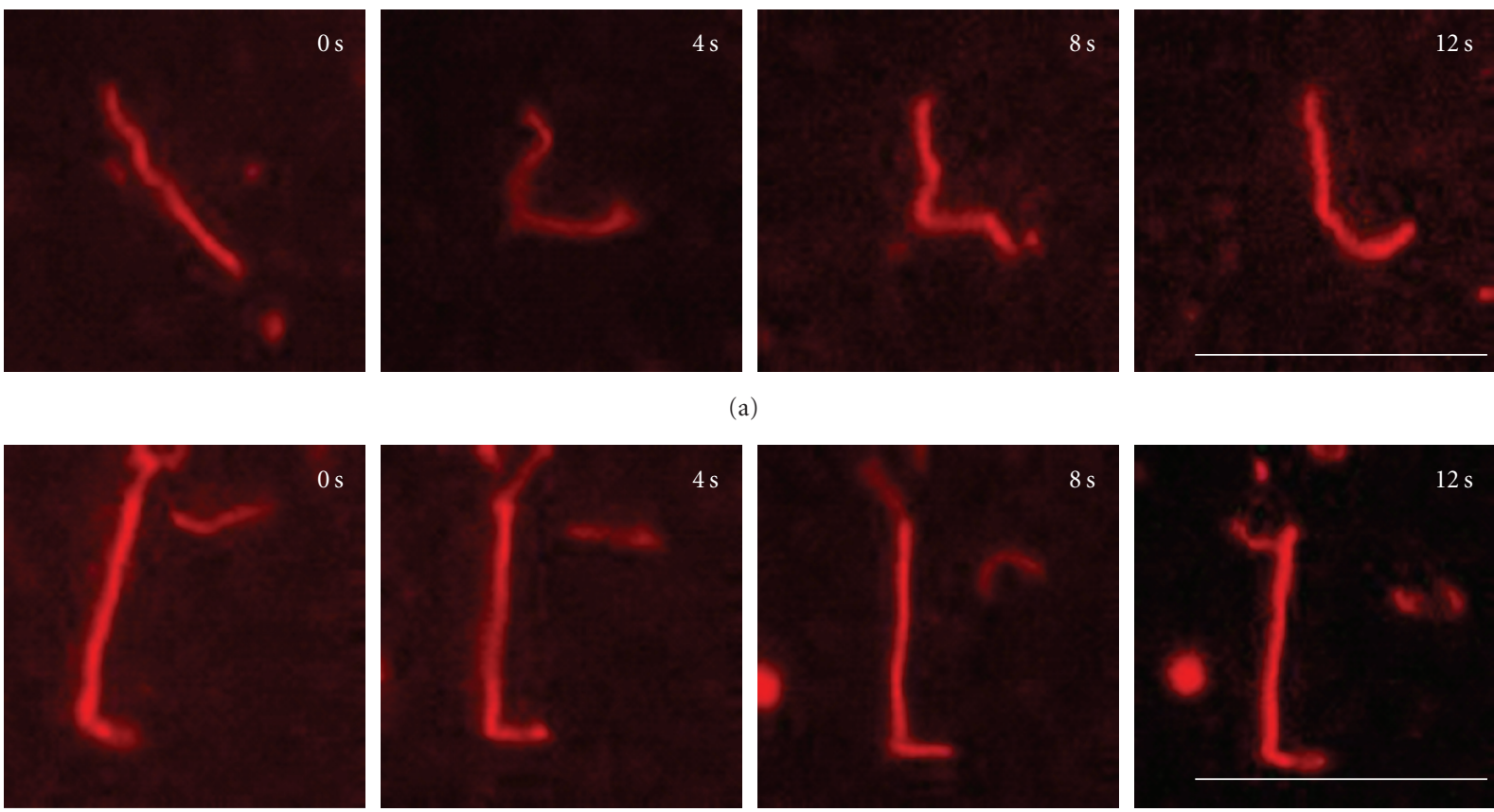

(b)

FIgURE 3: Dynamics of worm micelles from time-lapse fluorescence microscopy. Time-lapse fluorescence microscopy images of a flexible (a) and rigid (b) PCL-b-PEO worm micelle incorporating 5\% PCL-rhodamine is shown. Scale bar is $10 \mu \mathrm{m}$. 


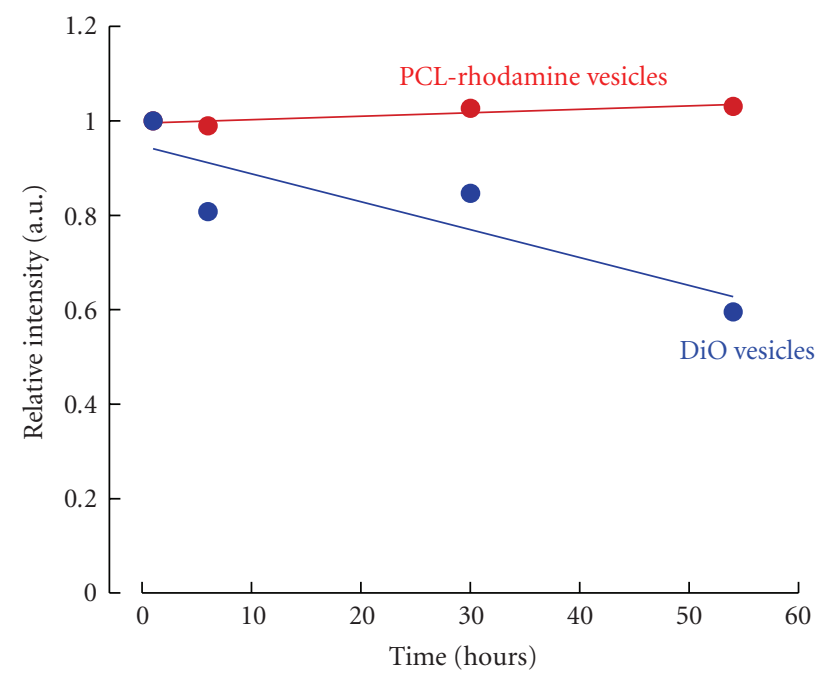

(a)

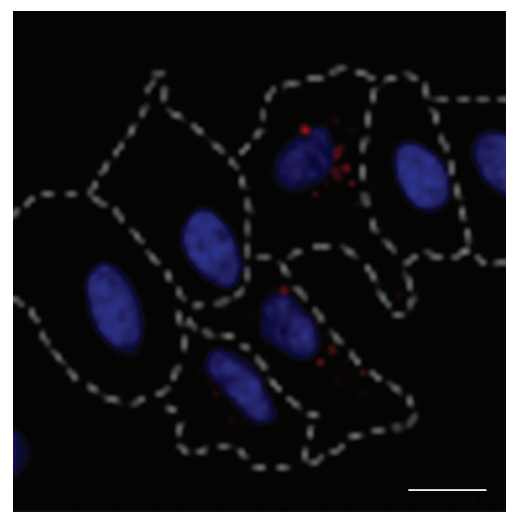

(b)

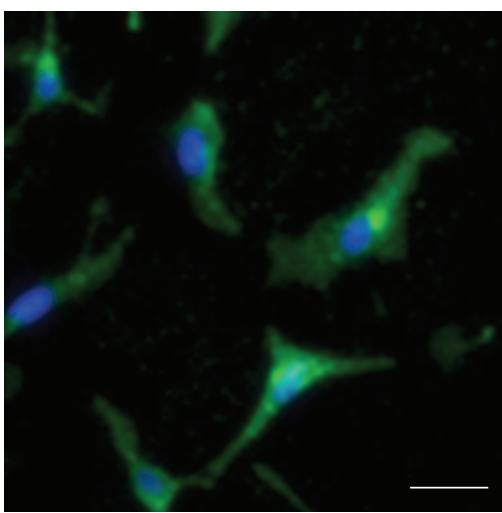

(c)

FIgURE 4: Dye retention and intracellular tracking of polymer assemblies. (a) The plot shows the retention and release of fluorophores from PCL-b-PEO assemblies as a function of time. While PCL-rhodamine shows no release, DiI is gradually released with time. (b) Covalent conjugation of dyes to polymers enables their in vivo tracking via fluoroscence microscopy. The image shows cell internalization of PCL-bPEO vesicles incorporating 5\% PCL-rhodamine. The cell membrane is indicated with broken white lines and the vesicles are shown in red. (c) When the lipophilic dye, DiI, is used for labeling PCL-b-PEO vesicles, it is transferred to the cell membranes (green). Scale bar is $10 \mu \mathrm{m}$.

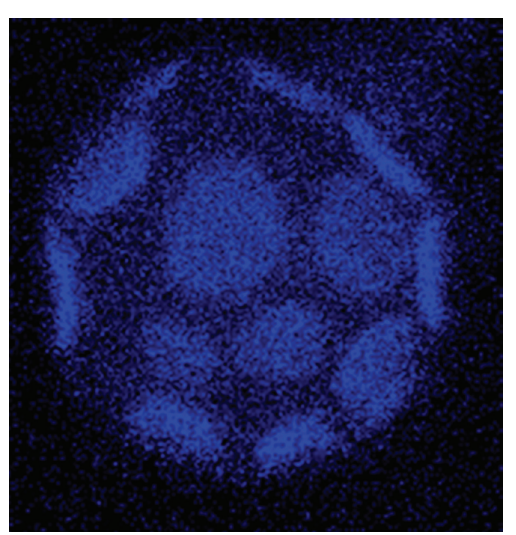

(a)

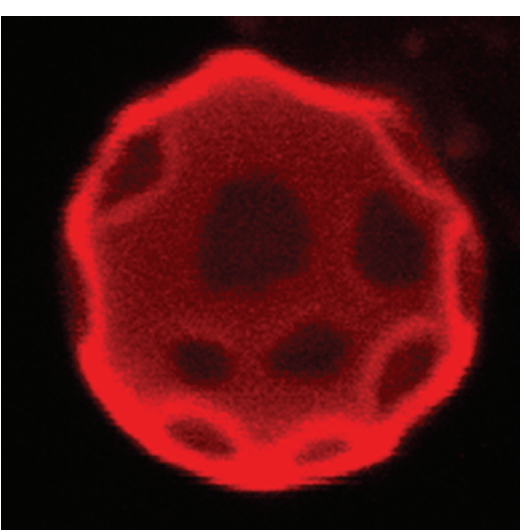

(b)

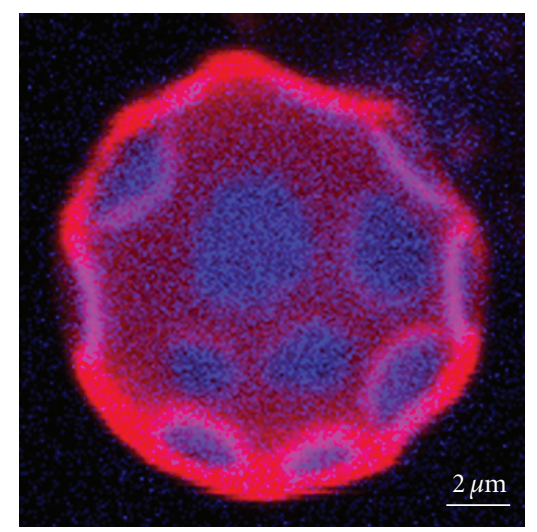

(c)

FIGURE 5: Visualization of phase separation in polymer assemblies. Vesicles prepared from cascade blue conjugated PBD-b-PAA (blue) and TMRCA-conjugated PBD-b-PEO (red) show phase separation in the presence of calcium at high pH conditions. Scale bar is $2 \mu \mathrm{m}$. 


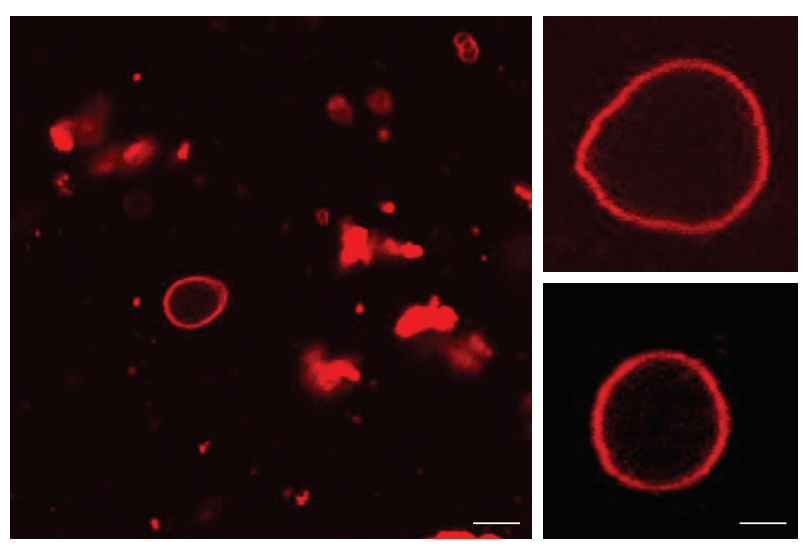

(a)

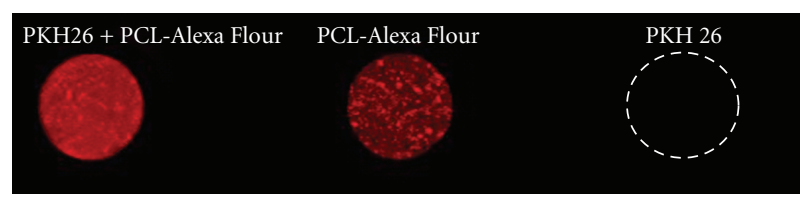

(b)

FIGURE 6: Near IR polymersomes. (a) Conjugation of AlexaFlour750 to PCL enables visualization in the near IR region $(748 \mathrm{~nm}$ excitation). The figure shows fluorescence microscopy images of PCL-b-PEO vesicles incorporating 5\% PCL-Alexa Flour. (b) The vesicles can also be imaged using Licor Odyssey near-IR imager where the dye was excited using a $800 \mathrm{~nm}$ laser. As a control, when PKH26-labeled vesicles was excited at $800 \mathrm{~nm}$ and no fluorescence was observed. Scale bar is $10 \mu \mathrm{m}$.

depends on the molecular weight, it is likely to be above the ambient conditions under which the worm micelles were imaged. However, flexibility to rigidity transitions in polymeric assemblies can be identified and characterized using time-lapse fluorescence microscopy.

\subsection{Dye Retention and Intracellular Tracking of Polymer} Assemblies. Covalent conjugation of dyes to amphiphilic polymers is useful for investigating the intra-cellular transport of polymer assemblies. This is particularly important when the externally added dye has a tendency to preferentially transfer to the cell membranes [24]. To investigate this, PCL-b-PEO vesicles incorporating 5\% PCL-rhodamine and labeled with $\mathrm{DiO}$ were prepared separately and the release of the dye was monitored as a function of time. The plot in Figure 4(a) shows that PCL-rhodamine is retained within the vesicles whereas $\mathrm{DiO}$ is released gradually with time. The labeled vesicles were then added to A549 human lung carcinoma cells and incubated for at least 12 hours. Figure 4(b) shows the images of cells incubated with PCLrhodamine vesicles after 24 hours and $4 \mathrm{~B}$ shows DiO-labeled vesicles after incubation for 12 hours. While PCL-rhodamine labeled vesicles (shown in red) do not show any labeling of the membranes and fluorescence is confined within the vesicles, the $\mathrm{DiO}$ dye transfers and labels the cell membranes. This clearly suggests that covalent conjugation of dye is important for intracellular tracking of polymer vesicles and worm micelles.

3.6. Visualization of Phase Separation in Polymer Assemblies. Phase separation between immiscible polymers in polymer assemblies like vesicles and wormlike micelles has been shown to occur from the nanoscale to the micron-scale. Most work has focused on immiscibility of the hydrophobic blocks as a driving force for phase separation. In these studies, phase separation occurs on the nano-scale, which requires the use of cryo-TEM and differences in the electron density of the two polymers to be visualized. More recently, ligand-induced phase separation has been observed between amphiphilic diblocks with similar hydrophobic blocks and dissimilar hydrophilic blocks (PBD-b-PEO and PBD-b-PAA) in the presence of a hydrophilic ligand (here, divalent calcium) [27]. In images taken by cryo-TEM, the hydrophilic blocks of these assemblies is difficult to image. To determine the distribution of each diblock species within these assemblies, fluorescence microscopy was used and a different fluorescent label was conjugated to each type of diblock. Phase separation in two-color polymersomes formed by film rehydration in the presence of calcium was imaged by laser scanning confocal microscopy (LSCM) (Figure 5). Distribution of PBD-b-PAA labeled with Cascade Blue was imaged using a $405 \mathrm{~nm}$ excitation laser source and seen Figure 5(a) as a confocal z-stack of one hemisphere of the polymersome. The distribution of PBD-b-PEO labeled with tetramethylrhodamine was imaged using a $543 \mathrm{~nm}$ laser excitation source as seen in Figure 5(b). A two-color overlay of the z-stacks (Figure 5(c)) clearly shows the lateral segregation of the two polymers within the polymersome assembly.

3.7. Near-IR Emissive Polymersomes. Polymersomes (polymer vesicles) encapsulate an aqueous lumen and typically range in size from a few hundred nanometers to a few tens of microns in diameter. The utility of these assemblies for drug delivery applications can be enhanced by tracking their biodistribution in vivo in live mice. Towards this goal, polymersomes labeled with near infrared emissive multiporphyrins [28], or lipophilic dyes as fluorophores can be used to track drug carriers or cells in live mice over time. Alternatively, Alexa flour C5 750 maleimide can be directly conjugated to thiol-terminated polycaprolactone and integrated within PCL-b-PEO vesicles. Figure 6(a) shows PCL-b-PEO vesicles incorporating 5\% PCL-Alexa Fluor imaged using an LSCM with a $748 \mathrm{~nm}$ laser excitation source. As a proof of principle, solutions containing these PCLAlexa Fluor 750 labeled vesicles were imaged in a 96 well plate on the Licor Odyssey using the $800 \mathrm{~nm}$ laser excitation source. Prior to imaging on the Odyssey, a red lipophilic dye PKH26 was added to the solution to confirm the presence of vesicles by epifluorescence microscopy. Solutions containing PCL-Alexa Fluor 750 labeled vesicles provided measurable fluorescence intensity when excited by the $800 \mathrm{~nm}$ laser on the Odyssey, which has been used in previous studies to image and quantify biodistribution in live animals and in freshly excised organs [29]. 


\section{Conclusions}

We have shown that polymeric wormlike micelles and polymersomes can be made fluorescent by covalently conjugating specific fluorophores. Commercially available fluorescent dyes are readily attached to diblock copolymers using simple conjugation chemistries. In addition to assessing the morphologies, covalent conjugation of dyes to diblock copolymers enables visualization of phase separation within assemblies and allows assessment of the fluctuation dynamics of worm micelles in real time. Covalent conjugation is particularly useful for the intracellular tracking of polymersomes. Finally, labeling with a near-infrared emissive dye (e.g., Alexa flour 750) provides new opportunity for tracking these assemblies in live rodents to better understand the in vivo behavior of these novel drug delivery and imaging vehicles.

\section{References}

[1] D. E. Discher and A. Eisenberg, "Polymer vesicles," Science, vol. 297, no. 5583, pp. 967-973, 2002.

[2] E. B. Zhulina, M. Adam, I. LaRue, S. S. Sheiko, and M. Rubinstein, "Diblock copolymer micelles in a dilute solution," Macromolecules, vol. 38, no. 12, pp. 5330-5351, 2005.

[3] P. Alexandridis and B. Lindman, Eds., Editors Amphiphilic Block Copolymers: Self-Assembly and Applications, Elsevier, New York, NY, USA, 2000.

[4] Y. Geng, P. Dalhaimer, S. Cai, et al., "Shape effects of filaments versus spherical particles in flow and drug delivery," Nature Nanotechnology, vol. 2, no. 4, pp. 249-255, 2007.

[5] Y. Geng and D. E. Discher, "Visualization of degradable worm micelle breakdown in relation to drug release," Polymer, vol. 47, no. 7, pp. 2519-2525, 2006.

[6] D. A. Christian, S. Cai, D. M. Bowen, Y. Kim, J. D. Pajerowski, and D. E. Discher, "Polymersome carriers: from self-assembly to siRNA and protein therapeutics," European Journal of Pharmaceutics and Biopharmaceutics, vol. 71, no. 3, pp. 463474, 2009.

[7] Y. Kim, M. Tewari, J. D. Pajerowski, et al., "Polymersome delivery of siRNA and antisense oligonucleotides," Journal of Controlled Release, vol. 134, no. 2, pp. 132-140, 2009.

[8] K. Yu and A. Eisenberg, "Multiple morphologies in aqueous solutions of aggregates of polystyrene-block-poly(ethylene oxide) diblock copolymers," Macromolecules, vol. 29, no. 19, pp. 6359-6361, 1996.

[9] S. Jain and F. S. Bates, "On the origins of morphological complexity in block copolymer surfactants," Science, vol. 300, no. 5618, pp. 460-464, 2003.

[10] E. J. G. Peterman, H. Sosa, and W. E. Moerner, "Singlemolecule fluorescence spectroscopy and microscopy of biomolecular motors," Annual Review of Physical Chemistry, vol. 55, pp. 79-96, 2004.

[11] J. W. Lichtman and J.-A. Conchello, "Fluorescence microscopy," Nature Methods, vol. 2, no. 12, pp. 910-919, 2005.

[12] J. Rao, A. Dragulescu-Andrasi, and H. Yao, "Fluorescence imaging in vivo: recent advances," Current Opinion in Biotechnology, vol. 18, no. 1, pp. 17-25, 2007.

[13] G. T. Hermanson, Ed., Bioconjugate Techniques, Academic Press, San Diego, Calif, USA, 1995.
[14] H. Shen and A. Eisenberg, "Morphological phase diagram for a ternary system of block copolymer $\mathrm{PPS}_{310}-b-\mathrm{PAA}_{52} /$ Dioxane $/ \mathrm{H}_{2} \mathrm{O}$," Journal of Physical Chemistry B, vol. 103, no. 44, pp. 9473-9487, 1999.

[15] S. Jain and F. S. Bates, "Consequences of nonergodicity in aqueous binary PEO-PB micellar dispersions," Macromolecules, vol. 37, no. 4, pp. 1511-1523, 2004.

[16] Y.-Y. Won, A. K. Brannan, H. T. Davis, and F. S. Bates, "Cryogenic transmission electron microscopy (cryo-TEM) of micelles and vesicles formed in water by poly(ethylene oxide)based block copolymers," Journal of Physical Chemistry B, vol. 106, no. 13, pp. 3354-3364, 2002.

[17] H. Cui, Z. Chen, S. Zhong, K. L. Wooley, and D. J. Pochan, "Block copolymer assembly via kinetic control," Science, vol. 317, no. 5838, pp. 647-650, 2007.

[18] Y. Geng and D. E. Discher, "Hydrolytic degradation of poly(ethylene oxide)-block-polycaprolactone worm micelles," Journal of the American Chemical Society, vol. 127, no. 37, pp. 12780-12781, 2005.

[19] P. Dalhaimer, H. Bermudez, and D. E. Discher, "Biopolymer mimicry with polymeric wormlike micelles: molecular weight scaled flexibility, locked-in curvature, and coexisting microphases," Journal of Polymer Science, Part B, vol. 42, no. 1, pp. 168-176, 2003.

[20] Y. Geng, F. Ahmed, N. Bhasin, and D. E. Discher, "Visualizing worm micelle dynamics and phase transitions of a charged diblock copolymer in water," Journal of Physical Chemistry B, vol. 109, no. 9, pp. 3772-3779, 2005.

[21] P. Dalhaimer, F. S. Bates, and D. E. Discher, "Single molecule visualization of stable, stiffness-tunable, flow-conforming worm micelles," Macromolecules, vol. 36, no. 18, pp. 68736877, 2003.

[22] S. Cai, K. Vijayan, D. Cheng, E. M. Lima, and D. E. Discher, "Micelles of different morphologies advantages of worm-like filomicelles of PEO-PCL in paclitaxel delivery," Pharmaceutical Research, vol. 24, no. 11, pp. 2099-2109, 2007.

[23] P. L. Soo, L. Luo, D. Maysinger, and A. Eisenberg, "Incorporation and release of hydrophobic probes in biocompatible polycaprolactone-block-poly(ethylene oxide) micelles: implications for drug delivery," Langmuir, vol. 18, no. 25, pp. 999610004, 2002.

[24] H. Chen, S. Kim, L. Li, S. Wang, K. Park, and J.-X. Cheng, "Release of hydrophobic molecules from polymer micelles into cell membranes revealed by Förster resonance energy transfer imaging," Proceedings of the National Academy of Sciences of the United States of America, vol. 105, no. 18, pp. 6596-6601, 2008.

[25] L. Luo, J. Tam, D. Maysinger, and A. Eisenberg, "Cellular internalization of poly(ethylene oxide)-b-poly( $\varepsilon$-caprolactone) diblock copolymer micelles," Bioconjugate Chemistry, vol. 13, no. 6, pp. 1259-1265, 2002.

[26] S. Muthian, K. Nithipatikom, W. B. Campbell, and C. J. Hillard, "Synthesis and characterization of a fluorescent substrate for the $\mathrm{N}$ - arachidonoylethanolamine (anandamide) transmembrane carrier," Journal of Pharmacology and Experimental Therapeutics, vol. 293, no. 1, pp. 289-295, 2000.

[27] D. A. Christian, A. Tian, W. G. Ellenbroek, et al., "Spotted vesicles, striped micelles and Janus assemblies induced by ligand binding," Nature Materials, vol. 8, no. 10, pp. 843-849, 2009.

[28] P. P. Ghoroghchian, P. R. Frail, K. Susumu, et al., "Nearinfrared-emissive polymersomes: self-assembled soft matter 
for in vivo optical imaging," Proceedings of the National Academy of Sciences of the United States of America, vol. 102, no. 8, pp. 2922-2927, 2005.

[29] D. A. Christian, S. Cai, O. B. Garbuzenko, et al., "Flexible filaments for in vivo imaging and delivery: persistent circulation of filomicelles opens the dosage window for sustained tumor shrinkage," Molecular Pharmaceutics, vol. 6, no. 5, pp. 13431352, 2009. 

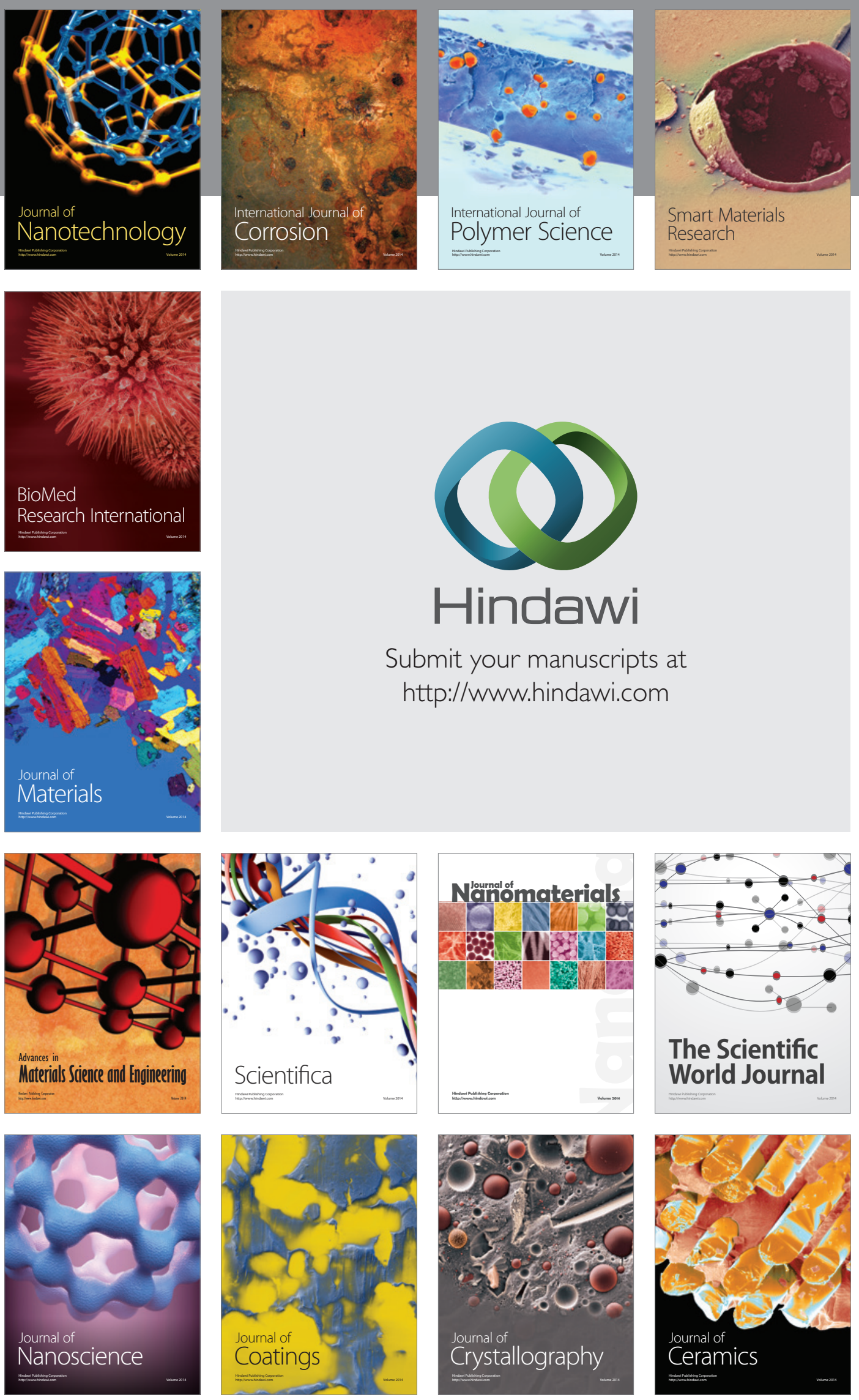

The Scientific World Journal

Submit your manuscripts at

http://www.hindawi.com

\section{World Journal}

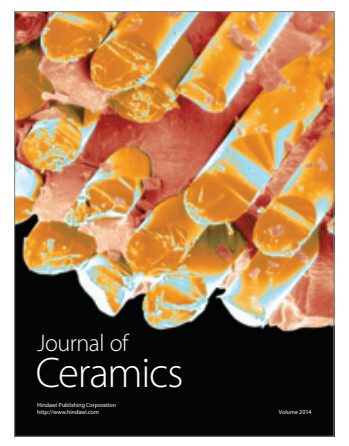

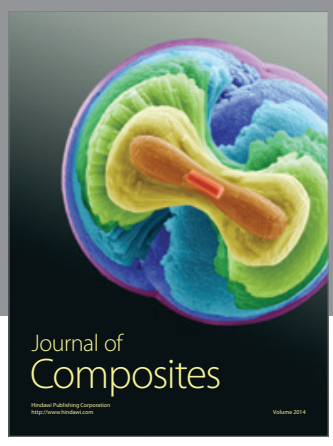
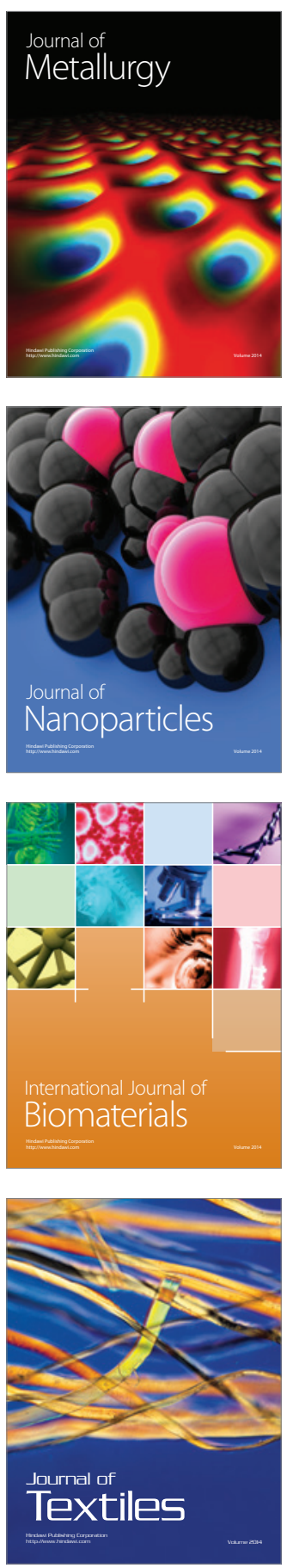